\title{
Optimization of Urban Water Supply Schemes based on Grey System Theory
}

\author{
Yun Bai ${ }^{1,2}$, Pu Wang ${ }^{1,2}$ and Jingjing Xie ${ }^{3}$ \\ ${ }^{1}$ Key Laboratory of Three Gorges Reservoir Region's Eco-Environment, Ministry of \\ Education, Chongqing University, Chongqing 400045, China. \\ ${ }^{2}$ National Centre for International Research of Low-carbon and Green Buildings, \\ Chongqing University, Chongqing 400045, China. \\ ${ }^{3}$ Testing Center for Science and Technology, Chongqing Academy of Science and \\ Technology, Chongqing 401123, China \\ yunbai@foxmail.com
}

\begin{abstract}
Urban water supply schemes are studied with multi-attribute characteristics taken into consideration. This paper proposes an optimized urban water supply scheme based on grey system theory. In the model, optimized indicators are given and their weights are acquired through optimized grey related analysis. These indicators are subject to standardization and are calculated to get grey incidence degree. The optimal water supply scheme is determined by the grey incidence degree. Finally, empirical test on the water supply scheme of a city is conducted to prove its objectivity, feasibility and reliability.
\end{abstract}

Keywords: Urban water supply; Scheme optimization; Grey system theory; Artificial intelligence

\section{Introduction}

With the development of technology and urbanization, people's life is improved and enriched. The expansion of urban increases the demand of industrial and household water. Therefore, it is important to take effective use of water resources, lower the water supply cost and improve the ecological environment. All these lay a key foundation for the sustainable development of the economy and the society $[1,2]$.

Therefore, it is significant to study the water supply scheme. However, when selecting the optimal scheme, overall factors involving with society, economy, ecology and environment need to be taken into consideration, including the reliability and quality of water supply, engineering layout, urban ecological benefits, energy consumption and cost. It is a system decision-making problem with multi-attribute. What's more, some factors are hard to be measured in a quantitative way. When addressing these fuzzy and uncertain factors, tradition and single economic analysis method fail to cope with the evaluation of the water supply scheme, not to mention provide information for social value and integrated benefit $[3,4]$. Therefore, this paper studies the optimized urban water supply scheme model based on grey system theory [5-7]. It establishes an optimized index system, acquires the weights, calculates grey incidence matrix and degree based on indicators and determines the optimal water supply scheme based on grey incidence degree. Grey incidence decision uses potential information when there is less data and uncertainties, ensuring the optimal water supply scheme to be objective and reasonable. 


\section{Optimized Indicators of Urban Water Supply Scheme and Grey Weight Distribution}

\subsection{Optimized Index System of Water Supply Scheme}

To select the optimal water supply scheme, social factors, economic factors, ecological and environmental factors all need to be considered. After consulting with experts and technicians in this field, this paper establishes an optimized index system with multi-attribute. First level index includes economic indicators, social indicators, ecological indicators and environmental indicators. Every first level index has its own second level index. Details are shown in Figure 1.

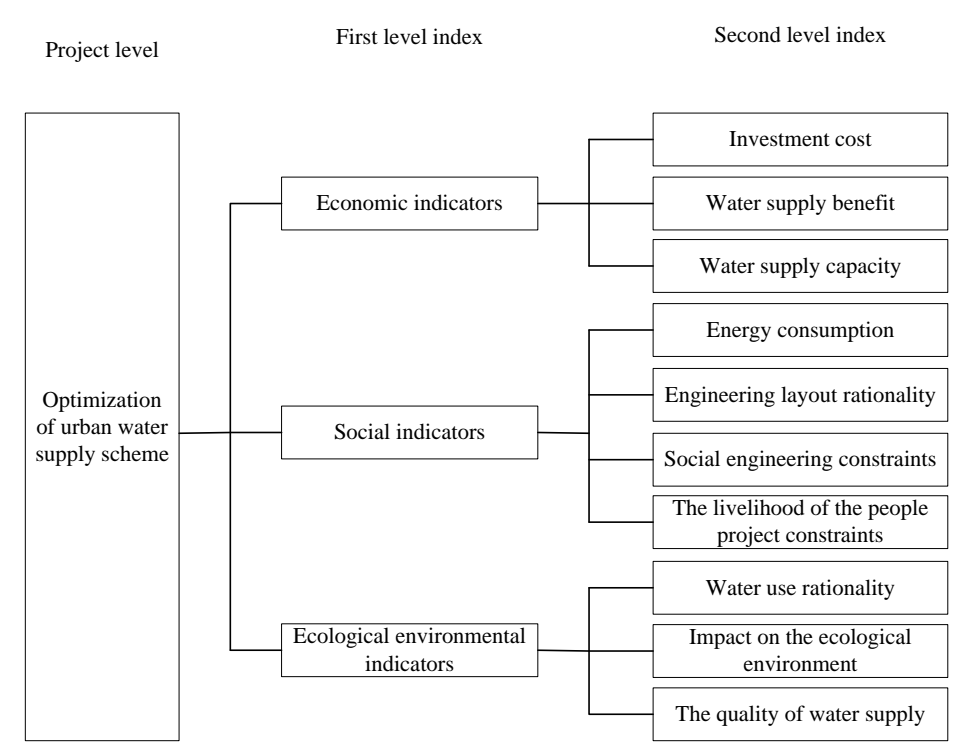

Figure 1. Optimized Index System of Water Supply Scheme

Suppose there are $m$ water supply schemes $S$. They constitute the multi-attribute decision set $\mathbf{S}=\left\{S_{1}, S_{2}, \ldots, S_{m}\right\}$, and $\mathbf{I}=\left\{I_{1}, I_{2}, \ldots, I_{n}\right\}$ refers to the set of indicators for the optimization. Then the characteristic value of scheme $S_{j}$ to optimized indicator $I_{j}$ is $a_{i j}(i=1,2, \ldots, m ; j=1,2, \ldots, n) . \mathbf{A}=\left(a_{i j}\right)_{\mathrm{mxn}}$ is described as the optimized decision matrix of scheme set $\mathbf{S}$ about optimized indicator set $\mathbf{I}$.

\subsection{Weight of Optimized Indicators of Water Supply Scheme}

Suppose there are $n$ optimized indicators $I, q$ experts are invited to score those indicators by the scale of $1 \sim 9$. And the scale sequence of optimized indicators is $Y_{j}=\left(y_{j}(1), y_{j}(2), \mathrm{L}, y_{j}(q)\right) .(j=1,2, \mathrm{~L}, n)$

Establish the grey incidence sequence of optimized indicators:

$$
Y_{0}=\left(y_{0}(1), y_{0}(2), \mathrm{L}, y_{0}(Q)\right),
$$

where $y_{0}(k)=\max _{1 \leq k \leq q}\left(y_{j}(k)\right)$.

The incidence coefficient between the optimized indicator sequence $Y_{i}$ and ideal indicator sequence $Y_{0}$ at $j$ is 


$$
\xi_{i}^{j}=\frac{\min _{j} \min _{k}\left|y_{0}(j)-y_{j}(k)\right|+\beta \max _{j} \max _{k}\left|y_{0}(j)-y_{j}(k)\right|}{\left|y_{0}(j)-y_{j}(k)\right|+\beta \max _{j} \max _{k}\left|y_{0}(j)-y_{j}(k)\right|},
$$

where $\beta$ refers to identification coefficient $(\beta=0.5)$.

The incidence degree between the optimized indicator sequence $Y_{i}$ and ideal indicator sequence $Y_{0}$ is:

$$
\delta(i)=\left(\sum_{k=1}^{q} \xi_{i}^{j}\right) / q
$$

Then the weight of the optimized indicator $I_{j}$ is expressed as

$$
w_{I_{j}}=\delta(j) /\left(\sum_{j=1}^{n} \delta(j)\right) \text {. }
$$

Here comes the weight sequence of all optimized indicators $\mathbf{W}=\left(w_{I_{1}}, w_{I_{2}}, L, w_{I_{n}}\right)^{\mathrm{T}}$.

\section{Optimized Model of Water Supply Scheme Based on Grey System Theory}

\subsection{Standardization of Optimized Indicators}

Under the optimized indicator system, optimized indicators vary in scale and quantity and are of different types. Some are of efficiency type that asks for a bigger indicator. Some are of cost type that asks for a smaller indicator. Some are of accurate quantitative indicators, some are of fuzzy and uncertain qualitative indicators, and others are of interval type. Thus, these indicators need standardization and normalization to eliminate their differentials. This paper divides indicators into three categories: cost type, efficiency type and interval type.

When the optimized indicator is of cost type, the value of $S_{i}$ to $I_{j}$ is $a_{i j}$, after standardization, the value $\bar{a}_{i j}$ is expressed as

$$
\bar{a}_{i j}=\frac{\max _{1 \leq i \leq m}\left(a_{i j}\right)-a_{i j}}{\max _{1 \leq i \leq m}\left(a_{i j}\right)-\min _{1 \leq i \leq m}\left(a_{i j}\right)} .
$$

When the optimized indicator is of efficiency type, the value of $S_{i}$ to $I_{j}$ is $a_{i j}$, after standardization, the value $\bar{a}_{i j}$ is expressed as

$$
\bar{a}_{i j}=\frac{a_{i j}-\min _{1 \leq i \leq m}\left(a_{i j}\right)}{\max _{1 \leq i \leq m}\left(a_{i j}\right)-\min _{1 \leq i \leq m}\left(a_{i j}\right)} .
$$

When the optimized indicator is of interval type, the value of $S_{i}$ to $I_{j}$ is $a_{i j}$, after standardization, the value $\bar{a}_{i j}$ is expressed as 


$$
\bar{a}_{i j}= \begin{cases}1-\frac{2\left(a-a_{i j}\right)}{a-\min _{1 \leq i \leq m}\left(a_{i j}\right)} & a_{i j} \leq a \\ 1 & a_{i j} \in[a, b] . \\ 1-\frac{2\left(a_{i j}-b\right)}{\max _{1 \leq i \leq m}\left(a_{i j}\right)-b} & a_{i j} \geq b\end{cases}
$$

\subsection{Analysis of Grey Incidence Degree}

Grey incidence degree analysis aims at studying the curve trend of data sequence based on grey system theory. The grey incidence degree of data sequence is introduced when there are few data and when the data are uncertain. Potential grey information is used for system prediction and decision [8-10]. Tradition system prediction or decision only considers a single incidence degree between effectiveness evaluation vectors of all system schemes and ideal effectiveness evaluation vector. But this method may not tab the information of system prediction or decision to the full use.

This is usually the case in the optimization of water supply scheme. When some scheme is closest to the ideal best scheme, it may also be not far from the ideal worst scheme. This makes the single incidence degree limited. Therefore, in the course of optimization, the grey incidence degree of effectiveness evaluation vectors of all system schemes and ideal best effectiveness evaluation vector need to be addressed. Otherwise, the optimized scheme cannot be objective and reliable.

Assume that all optimized indicators are standardized and of efficiency type. The ideal best water supply scheme $S_{O}$ and the ideal worst water supply scheme $S_{N}$ is described as

$$
\begin{gathered}
S_{O}=\left(\bar{a}_{1}^{O}, \bar{a}_{2}^{O}, \ldots, \bar{a}_{n}^{O}\right)=\left(\max _{1 \leq i \leq m}\left(\bar{a}_{i 1}\right), \max _{1 \leq i \leq m}\left(\bar{a}_{i 2}\right), \ldots, \max _{1 \leq i \leq m}\left(\bar{a}_{i n}\right)\right), \\
S_{N}=\left(\bar{a}_{1}^{N}, \bar{a}_{2}^{N}, \ldots, \bar{a}_{n}^{N}\right)=\left(\min _{1 \leq i \leq m}\left(\bar{a}_{i 1}\right), \min _{1 \leq i \leq m}\left(\bar{a}_{i 2}\right), \ldots, \min _{1 \leq i \leq m}\left(\bar{a}_{i n}\right)\right) .
\end{gathered}
$$

According to grey incidence analysis theory, the incidence coefficient $\varphi_{i j}^{O}$ between water supply scheme $S_{i}$ and the ideal optimal water supply scheme $S_{O}$ about indicator $I_{j}$ is

$$
\varphi_{i j}^{O}=\frac{\min _{i} \min _{j}\left|\bar{a}_{i j}-\bar{a}_{j}^{O}\right|+\beta \max _{i} \max _{j}\left|\bar{a}_{i j}-\bar{a}_{j}^{O}\right|}{\left|\bar{a}_{i j}-\bar{a}_{j}^{O}\right|+\beta \max _{i} \max _{j}\left|\bar{a}_{i j}-\bar{a}_{j}^{O}\right|} .
$$

The incidence coefficient $\varphi_{i j}^{N}$ between water supply scheme $S_{i}$ and the ideal worst supply scheme $S_{N}$ about indicator $I_{j}$ is

$$
\varphi_{i j}^{N}=\frac{\min _{i} \min _{j}\left|\bar{a}_{i j}-\bar{a}_{j}^{N}\right|+\beta \max _{i} \max _{j}\left|\bar{a}_{i j}-\bar{a}_{j}^{N}\right|}{\left|\bar{a}_{i j}-\bar{a}_{j}^{N}\right|+\beta \max _{i} \max _{j}\left|\bar{a}_{i j}-\bar{a}_{j}^{N}\right|} .
$$

Given different weight of optimized indicators, the weighed grey incidence degree $\psi_{i}^{O}$ between the supply scheme $S_{i}$ and the ideal best water supply scheme $S_{O}$ about all indicators is 


$$
\psi_{i}^{O}=\sum_{j=1}^{n}\left(w_{I_{I}} \times \varphi_{i j}^{O}\right) .
$$

The weighed grey incidence degree $\psi_{i}^{N}$ between the supply scheme $S_{i}$ and the ideal worst water supply scheme $S_{N}$ about all indicators is

$$
\psi_{i}^{N}=\sum_{j=1}^{n}\left(w_{I_{I}} \times \varphi_{i j}^{N}\right) .
$$

Through analysis, the bigger the weighed grey incidence degree $\psi_{i}^{O}$ is, the closer $S_{i}$ is to $S_{O}$ and the better the scheme will be. The smaller the weighed grey incidence degree $\psi_{i}^{N}$ is, the closer $S_{i}$ is to $S_{N}$ and the worse the scheme will be. So, the grey incidence degree $\psi_{i}$ is defined as

$$
\psi_{i}=\frac{\left(\psi_{i}^{O}\right)^{2}}{\left(\psi_{i}^{O}\right)^{2}+\left(\psi_{i}^{N}\right)^{2}} .
$$

$\psi_{i}$ is useful to get the optimal water supply scheme.

\subsection{Optimal Model and Algorithm}

By establishing the optimized index system of water supply scheme and standardization and normalization of indicators, an integrated grey incidence degree is acquired. The algorithm is described as follows:

Step 1 Consult with experts and technicians to get the multi-attribute optimized index system;

Step 2 Invite experts and technicians to score optimized indicators with the scale of $1 \sim 9$ and get different weights based on expression (1) to (4);

Step 3 Acquire water supply scheme based on urban water demand and from the scheme set;

Step 4 Based on the scheme set, apply optimized indicators to standardization and normalization based on expression (5), (6) and (7);

Step 5 Construct the ideal best water supply scheme $S_{O}$ and the ideal worst water supply scheme $S_{N}$ based on expression (8) and (9);

Step 6 Get the grey incidence coefficient between different water supply schemes and the ideal best water supply scheme $S_{O}$ and the ideal worst water supply scheme $S_{N}$ based on expression (10) and (11);

Step 7 Get the grey incidence degree between different water supply schemes and the ideal best water supply scheme $S_{O}$ and those and the ideal worst water supply scheme $S_{N}$ based on expression (12) and (13);

Step 8 Get the integrated grey incidence degree between different water supply schemes and the ideal best water supply scheme based on expression (14);

Step 9 Rank the schemes according to the integrated grey incidence degree. The one with the largest integrated grey incidence degree is the optimal scheme.

\section{Case Study}

This paper tests the model and algorithm based on a water supply scheme of a city. Under the optimized index system in Chapter 2.1, weights of optimized indicators are 
given by experts. The weight of first level index and second level index is shown in Table 1 and Table 2.

Table 1. Weight of First Level Index

\begin{tabular}{ccc}
\hline First level index & Weight & Expert score \\
\hline Economic index & 0.356 & $9,8,9,9,7,8$ \\
Social index & 0.312 & $8,7,8,8,7,9$ \\
Ecological and environmental index & 0.337 & $9,8,7,8,8,8$ \\
\hline
\end{tabular}

Table 2. Weight of Second Level Index

\begin{tabular}{cccc}
\hline First level index & Second level index & Weight & Expert score \\
\hline \multirow{3}{*}{ Economic index } & Investment cost & 0.332 & $9,8,9,9,7,9$ \\
& Water supply benefit & 0.323 & $8,9,8,9,9,9$ \\
& Water supply capacity & 0.340 & $9,9,9,9,9,9$ \\
& $\begin{array}{c}\text { Energy consumption } \\
\text { Engineering layout }\end{array}$ & 0.259 & $9,8,9,9,9,9$ \\
& $\begin{array}{c}\text { rationality } \\
\text { Social index }\end{array}$ & 0.235 & $8,8,7,9,8,9$ \\
& $\begin{array}{c}\text { Social engineering } \\
\text { constraints }\end{array}$ & 0.253 & $8,8,8,7,8,9$ \\
& $\begin{array}{c}\text { The livelihood of the people } \\
\text { project constraints }\end{array}$ & 0.253 & $8,7,8,8,8,9$ \\
Ecological and & Water use rationality & 0.324 & $8,8,9,8,7,8$ \\
environmental index & Impact on the ecological & 0.282 & $8,9,7,7,7,8$ \\
& $\quad$ environment & 0.394 & $9,9,9,9,9,8$ \\
\hline
\end{tabular}

After survey and analysis of this particular water supply scheme, there come three water supply schemes. The parameters of each one are shown in Table 3.

Table 3. Parameters of Water Supply Scheme

\begin{tabular}{cccc}
\hline Optimized index & Scheme 1 & $\begin{array}{c}\text { Parameters } \\
\text { Scheme } 2\end{array}$ & Scheme 1 \\
\hline Investment cost $\left(\right.$ Yuan/ $\left./ V^{3}\right)$ & 1.21 & 1.17 & 1.22 \\
Water supply benefit(Yuan/ $\left.V^{3}\right)$ & 1.19 & 1.05 & 1.12 \\
Water supply capacity & 80 & 80 & 80 \\
Energy consumption & 0.85 & 0.75 & 0.75 \\
Engineering layout rationality & 0.90 & 0.85 & 0.90 \\
Social engineering constraints & 0.6 & 0.7 & 0.6 \\
The livelihood of the people project & 0.5 & 0.5 & 0.6 \\
constraints & 0.8 & 0.8 & 0.9 \\
Water use rationality & 0.5 & 0.6 & 0.5 \\
Impact on the ecological environment & 0.8 & 0.8 & 0.85 \\
The quality of water supply & & &
\end{tabular}

After standardization, parameters for three water supply schemes are shown in Table 4. 
Table 4. Parameters of Water Supply Scheme After Standardization

\begin{tabular}{|c|c|c|c|}
\hline \multirow{2}{*}{ Optimized index } & \multicolumn{3}{|c|}{ Parameters } \\
\hline & Scheme 1 & Scheme 2 & Scheme 3 \\
\hline Investment cost & 0.20 & 1.00 & 0 \\
\hline Water supply benefit & 1.00 & 0 & 0.50 \\
\hline Water supply capacity & 1.00 & 1.00 & 1.00 \\
\hline Energy consumption & 0 & 1.00 & 1.00 \\
\hline Engineering layout rationality & 1.00 & 0 & 1.00 \\
\hline Social engineering constraints & 1.00 & 0 & 1.00 \\
\hline $\begin{array}{l}\text { The livelihood of the people project } \\
\text { constraints }\end{array}$ & 1.00 & 1.00 & 0 \\
\hline Water use rationality & 0 & 0 & 1.00 \\
\hline Impact on the ecological environment & 1.00 & 0 & 1.00 \\
\hline The quality of water supply & 0 & 0 & 1.00 \\
\hline
\end{tabular}

Apply the grey incidence analysis algorithm to parameters after standardization and get three grey incidence degrees, as is shown in Table 5.

Table 5. Grey Incidence Degree of Water Supply Scheme

\begin{tabular}{cccc}
\hline Ideal scheme & \multicolumn{3}{c}{ Grey incidence degree } \\
& Scheme 1 & Scheme 2 & Scheme 3 \\
\hline The ideal best water supply scheme & 0.719 & 0.595 & 0.814 \\
The ideal worst water supply scheme & 0.594 & 0.737 & 0.485 \\
\hline
\end{tabular}

According to grey incidence degree model, get the integrated grey incidence sequence of three water supply scheme and the ideal best water supply scheme. Hence the optimal water supply scheme is scheme 3.

\section{Conclusion}

This paper proposes an optimized urban water supply scheme based on the grey system theory. In the model, the optimized indicators are given and their weights are acquired through the optimized grey related analysis. So the optimized index system is objective and reliable. These indicators are subject to standardization for calculating the grey incidence degree. The integrated grey incidence degree between the water supply scheme and the ideal best water supply scheme and the ideal worst water supply scheme is calculated to make the result more reliable. Finally, empirical test on the water supply scheme of a city is conducted to prove its feasibility and reliability. This model provides scientific support for urban water supply scheme as well as for its implementation.

\section{Acknowledgements}

This work is supported in part by the National Key Technology Research and Development Program of China (2012BAH32F01, 03), and the 111 Project (No.B13041). 


\section{References}

[1] S.J. Wu, J.H. Yoon, and R.G. Quimpo, "Capacity-weighted water distribution system reliability”, Reliability Engineering and System Safety, vol. 42, no. 1, (1993).

[2] K. Li, X.H. Chen, T. Jiang and X.J. Tu, "Application of multi-objective system fuzzy optimization theory to selecting table-water resources in Foshan City", Acta Scientiarum Naturalium Universitatis Sunyatseni, vol. 44, no. 4, (2005).

[3] W.H. Yang, L.F. Wang, K. Wang and L.J. Zhao, "Application of modified osculating value method in evaluation of water environmental quality", Journal of Water Resources and Water Engineering, vol. 16, no. 2, (2005).

[4] C.Y. Liu and B.H. Wang, "Application of artificial neural network on comprehensive evaluation of water conservancy project schemes", Water Conservancy Science and Technology and Economy, vol. 18, no. 6, (2012).

[5] Y.G. Dang, S.F. Liu, B. Liu and Y. Tao, "Study on grey incidence decision model of the dynamic multipleattribute", Engineering Science, vol. 7, no. 2, (2005).

[6] S.F. Liu, Z.G. Fang, Y. Yang and J. Forrest, "General grey numbers and their operations", Grey Systems: Theory and Application, vol. 2, no. 3, (2012).

[7] S.F. Liu, W.F. Yuan, K.Q. Sheng, "Multi-attribute intelligent grey target decision model", Control and Decision, vol. 25, no. 8, (2010).

[8] T.C. Wang, A.J. Yang and L.F. Bu, "Mechanism scheme design based on multi-attribute extension gray relevant optimized decision-making model”, Systems Engineering- Theory and Practice, vol. 33, no. 9, (2013).

[9] J.L. Deng, "Properties of relational space for grey system", Essential topics on grey system theory and applications, (1988), pp 1-13.

[10] S.F. Liu, Z.G. Fang and Y. Lin, "Study on a new definition of degree of grey incidence", Journal of Grey System, vol. 9, no. 2, (2006).

\section{Authors}

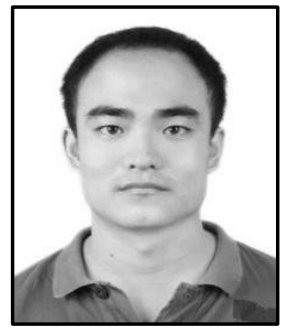

Yun Bai, currently, he is a Ph.D. candidate at School of Urban Construction and Environmental Engineering, Chongqing University, China. His research interests include municipal water supply scheduling and optimization.

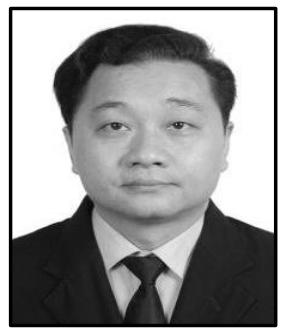

Pu Wang, currently, he is a Professor at School of Urban Construction and Environmental Engineering, Chongqing University, China. His research interests include municipal water supply scheduling and optimization.

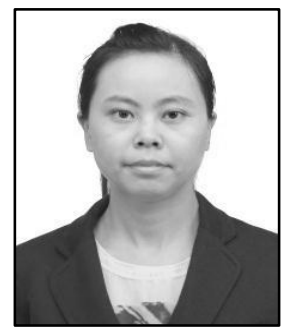

Jingjing Xie, she received the Master Degree from the Department of Chongqing Technology and Business University, China, in 2008. Currently, she is an Engineer at Chongqing Academy of Science and Technology, China. Her research interests include data mining and decision support system. 